\title{
Preview Control of Trajectory Tracking for Servo System Based on Optimal Preview Control Method
}

\author{
Mohammed M. Saleh All-bail ${ }^{1}$, Yuegang $\operatorname{Tan}^{1} \&$ Liang $\mathrm{Li}^{1}$ \\ ${ }^{1}$ School of Mechanical and Electronic Engineering, Wuhan University of Technology, Hubei, China \\ Correspondence: Mohammed M. Saleh All-bail, School of Mechanical and Electronic Engineering, Wuhan \\ University of Technology, 122 Luoshi Road, Wuhan 430070, Hubei, China. Tel: 86-189-7162-1020. E-mail: \\ malbeel@yahoo.com
}

Received: January 13, 2012

Accepted: February 2, $2012 \quad$ Online Published: May 24, 2012

doi:10.5539/mer.v2n1p18

URL: http://dx.doi.org/10.5539/mer.v2n1p18

This work is supported by Specialized Research Fund for the Doctoral Program of Higher Education (20100143110012)

\begin{abstract}
This paper researches on trajectory tracking control system through simulation, theory and experiment. It deals with the use of Universal Motion and Automation Controller UMAC based on preview control method to give good trajectory tracking performance of 3-axis servo system. The subject of this paper is to study and design space object trajectory tracking controller based on optimal preview method and then implement it in servo system.
\end{abstract}

Keywords: optimal preview control method, optimal control system, space object trajectory tracking, servo-system, UMAC

\section{Introduction}

The Optimal Preview Control Method OPCM is a new control theory in the recent years, which has been applied in many aspects ( $\mathrm{Li}$, Zhou, Hu, \& Hu, 2000) and ( $\mathrm{Li} \& \mathrm{Lu}, 2011)$. The field of Preview Control is correlated with using advanced knowledge of disturbances or references to improve the tracking quality or the disturbance rejection (Andrew Hazell, 2008). The Preview controller is a controller that uses the future information on the target signal and or disturbances in the design process. Generally, OPCM is based on the error, the difference between target signal and the actual trajectory. It is a useful method for the control, namely, Multiple Input Multiple Output MIMO control system. Optimal preview control method for servo systems is an effective to improve the phase response as compared to optimal control method. Once obtained the future information which depends on the activity of the control system, we will be able to calculate the evaluation function.

In (Chandeok \& Daniel, 2006), propose a novel approach for solving the optimal feedback control problem. They construct a Hamiltonian system by using the necessary conditions for optimality, and treat the resultant phase flow as a canonical transformation. Then starting from the Hamilton-Jacobi equations for generating functions they derive a set of 1st Order Quasi Linear partial differential equations with the relevant terminal conditions, which forms the well-known Cauchy problem. They have presented a new method for solving the optimal feedback control problem. While in (Andrew Hazell, 2008), the controller optimality is determined using either the $\mathrm{H}_{2}$ or $\mathrm{H}_{\infty}$ norms. The lateral tracking problem is first tackled in (Sharp \& Valtetsiotis, 2001), in the context of steering control of a road vehicle moving at a constant speed along a path. The control is designed using a linear model described in an inertial reference frame; however, as the vehicle moves, the reference frame is updated so that it is aligned to, and coincides with, the vehicle. This process is justified by the intuitive notion that a human driver controls a vehicle using a 'local' reference frame that is aligned to the vehicle. The algorithm is further supported by numerical studies that find the control to be invariant to both changes in position of the reference frame, and also small rotations (Sharp \& Valtetsiotis, 2001). Although, the preview theory of tracking control using the future information has been studied extensively since long time but did not get enough applications in the field of servo control systems, which is one of the most important techniques used in modern industrial processing, that will increase the accuracy and efficiency of the industrial product. Preview control 
offers one of the few means for improving tracking performance beyond that provided by causal feedback (Ren, Chen, hara, \& Qiu, 2001). The term "Preview Control" is usually associated with a particular class of anticipative control problems with a preview horizon that extends for a fixed time into the future. The objective of Optimal Preview Control is to find a controller that minimizes the power of the controlled output under definite assumptions about the reference and disturbance signals. Another objective is to determine the control signals that will cause a process to satisfy the physical constraints and at the same time minimize (or maximize) some performance criterion (Lewis \& Syrmos, 1995). The optimal preview control problems have been studied extensively throughout the 60's and 70's of the last century (Sheridan, 1966; Bender, 1968) and (Tomizuka, 1976). Before that, under normal conditions the servo control system did not use the future information. It is interested with using future information (advanced knowledge) of disturbances or references to minimize the evaluation function in the control period (optimal control theory) in order to improve tracking quality or disturbance rejection. The use of future information has great potential for performance improvement.

\section{Optimal Control with Preview Feedforward Compensation}

The following discrete-time control system is considered

$$
x(i+1)=A x(i)+B u(i)+E d(i) ; \quad y(i)=C x(i)+D u(i)
$$

Where: $x(i)$ is a $(\mathrm{n} \times 1)$ state vector, $y(\mathrm{i})$ is a $(m \times 1)$ output vector, $u(i)$ is a $(r \times 1)$ control vector, $d(i)$ is a $\mathrm{q} \times 1$ disturbance vector. While; matrices $A, B, C$, and $D$ are independent of (i) matrices with $(n \times n),(n \times r),(m \times n)$, and $(m \times r)$ dimensions respectively. Where: $R(i)$ is a $(\mathrm{m} \times 1)$ objective signal . The error signal is defined as:

$$
e(i)=R(i)-Y(i)
$$

Assume that system (1) is controllable and observable and there exists $r \geq m$. it is easy to obtain the following error system.

$$
X_{0}(i+1)=\Phi X_{0}(i)+G \Delta u(i)+G_{R} \Delta R(i+1)+G_{d} \Delta d(i)
$$

Where:

$$
\begin{aligned}
& X_{0}(i)=\left[\begin{array}{c}
e(i) \\
\Delta x(i)
\end{array}\right], \Phi=\left[\begin{array}{cc}
I_{m} & -C A \\
0 & A
\end{array}\right], G=\left[\begin{array}{c}
-C B \\
B
\end{array}\right], G_{R}=\left[\begin{array}{c}
I_{m} \\
0
\end{array}\right], G_{d}=\left[\begin{array}{c}
-C E \\
E
\end{array}\right], \\
& \Delta x(i)=x(i)-x(i-1), \quad \Delta u(i)=u(i)-u(i-1), \Delta R(i)=R(i)-R(i-1), \\
& \Delta d(i)=d(i)-d(i-1)
\end{aligned}
$$

We introduce the performance index for the system (1) as follows:

$$
J=\sum_{i=1}^{M_{R}}\left[x^{T}(i) Q x(i)+u^{T} H u(i)\right]
$$

Where: $Q$ and $H$ are weighting matrices settled by the designer.

Suppose the target value signal from the present time (i) to the future step $M_{R}$, as well as to the interference signal of step $M_{d}$ are all known. Based on previous research (Liao, Cui, \& Shen, 2009) and (Lin \& Brandt, 1998), the optimal control input with feedforward compensation of system (1) is achieved as the following:

$$
\Delta u(i)=F_{0} X_{0}+\sum_{j=1}^{M_{R}} F_{R}(j) \Delta R(i+j)+\sum_{j=0}^{M d} F_{d}(j) \Delta d(i+j)
$$

Where:

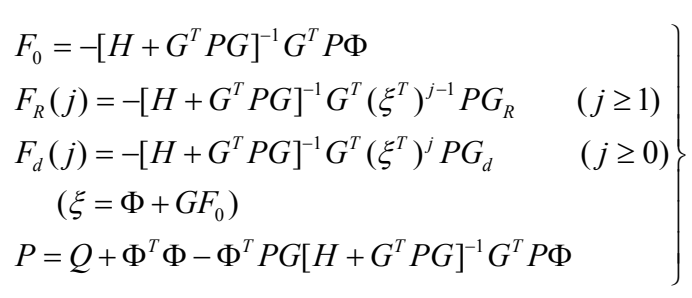


Here: $F_{0}$ is a state feedback matrix of the error system, $F_{R}$ is a feed-forward parameter matrix, $F_{d}$ is preview feed-forward parameter matrix for disturbance signal, and $P$ is The algebraic Riccati equation. The first item on the right side of Eq. 4 expresses the conventional optimal servo system, and based on this, the optimal preview servo system is composed of preview feed-forward compensation item by making use of future target value as expressed in the second item and preview feed-forward compensation item by making use of future interference as expressed in the third term. In addition, if the initial value is not zero, it also can carry out initial value compensation. Through feed-forward coefficient matrix, it can be seen that the feedback characteristic of optimal preview servo system is only determined by weight coefficients matrix $Q$ and $H$ of evaluation function, which only influences the feed-forward effect of target value signal and target signal.

\section{Relationship between Preview Steps and Evaluation Function}

Since, $\xi=\Phi+G F_{0}$ is the characteristic matrix of closed-loop system, the modules of all of its characteristic values should be less than 1, then, from the Eq. (5) it can be known:

$$
F_{R}(j+1)<F_{R}(j) \text { and } \lim _{j \rightarrow \infty} F_{R}(j)=0, F_{d}(j+1)<F_{d}(j) \text { and } \lim _{j \rightarrow \infty} F_{d}(j)=0
$$

That is to say, the more distant future information has less influence on the functional value of performance index; when the preview steps reach a certain extent, and if the preview steps are increased further, then the effect of functional value of performance index will not be obvious. The following theorem presents the relation between preview steps and functional value of system's performance index:

Theorem: with respect to error system assume that its target value and disturbance signal present step change respectively and independently, the target value from current time $(i)$ to future $M_{R}$ step as well as to the disturbance value of future $M_{d}$ step are known, and the optimal preview control input is expressed in Eq. (4), so then, the functional value of performance index specified in Eq. (3) will be monotonically decreased degree by degree along with the increase of preview steps, until reach to a fixed value, that is:

$$
\left.\begin{array}{l}
\lim _{M_{R} \rightarrow \infty} J_{R}=R_{0}^{T} G_{R}^{T}[P-P S P] G_{R} R_{0}=G_{R_{\infty}} \\
\lim _{M_{d} \rightarrow \infty} J_{d}=R_{0}^{T} G_{d}^{T}[P-P S P] G_{d} R_{0}=G_{d_{\infty}}
\end{array}\right\}
$$

Where: $J_{R}$ is the pre-viewable part of the evaluation function value, $J_{d}$ is the distributed part of the evaluation function, and $\mathrm{S}$ is positive definite symmetric matrix of the following Lyapunov formula

$$
\xi S \xi^{T}-S+G\left[H+G^{T} P G\right]^{-1} G^{T}=0
$$

The use of optimal preview servo implemented in the pre-viewable part of the evaluation function value predicted $J_{F R}$ and un-preview optimal predicted value of the servo system evaluation function measured $J_{R 0}$, then the preview evaluation function $J_{P R}$ can be obtained as following:

$$
J_{P R}=J_{R 0}-J_{R F}\left(M_{R}\right)
$$

Where:

$$
\begin{gathered}
J_{R 0}=R_{0}^{T} G_{R}^{T} P G_{R} R_{0} \\
J_{R F}\left(M_{R}\right)=\sum_{j=1}^{M_{R}} R_{0}^{T} G_{R}^{T} P \xi^{j-1} G\left[H+G^{T} P G\right]^{-1} G^{T}\left(\xi^{T}\right)^{j-1} P G_{R} R_{0}
\end{gathered}
$$

Compared with:

$$
J_{R F}\left(M_{R}+1\right)=J_{R F}\left(M_{R}\right)+\Delta J_{R F}\left(M_{R}+1\right)
$$

Where:

$$
\Delta J_{R F}\left(M_{R}+1\right)=R_{0}^{T} G_{R}^{T} P \xi^{M_{R}} G\left[H+G^{T} P G\right]^{-1} G^{T}\left(\xi^{T}\right)^{M_{R}} P G_{R} R_{0}
$$

Because $\left[H+G^{T} P G\right]^{-1}$ is positive definite matrix so $\Delta J_{R F}\left(M_{R}+1\right)>0$ can be known according to Eq. (8), and therefore it can be known according to Eq. (9) and Eq. (10): 


$$
J_{R}\left(M_{R}+1\right)<J_{R}\left(M_{R}\right)
$$

The above formula presents that $J_{R}\left(M_{R}\right)$ is monotone decreasing function. Then prove convergence. So that:

$$
S_{R}=\lim _{M_{R} \rightarrow \infty} \sum_{j=1}^{M_{R}} \xi^{j-1} G\left[H+G^{T} P G\right]^{-1} G^{T}\left(\xi^{T}\right)^{j-1}
$$

Is the following:

$$
\lim _{M_{R} \rightarrow \infty} R_{0}^{T} G_{R}^{T} P G_{R} R_{0}-\lim _{M_{R} \rightarrow \infty} J_{R F}=R_{0}^{T} G_{R}^{T} P G_{R} R_{0}-R_{0}^{T} G_{R}^{T} P S_{R} P G_{R} R_{0}
$$

And from (11) we can see that:

$$
S_{R}=G\left[H+G^{T} P G\right]^{-1} G^{-1}+\xi S \xi^{T}
$$

Which is satisfying Lyapunov equation specified in Eq. (7). And also because the solution of this equation is only one, therefore $S_{R}=S$, according to Eq. (1), it also can be known that Eq. (6) is true. Similarly, we can prove the condition previewed by disturbance.

In order to study the effective preview steps and to present the role of preview visually, it needs to calculate and implement the previewed evaluation function value $J_{R F}$ of preview part in the optimal preview system, then measure the comparative value with evaluation function value $J_{R 0}$ of optimal servo system without preview, this comparative value is calculated as follows:

$$
\frac{J_{R F}}{J_{R 0}}=\frac{\sum_{j=1}^{M_{R}} G_{R} P \xi^{j-1} G\left[H+G^{T} P G\right]^{-1} G^{T}\left(\xi^{T}\right)^{j-1} P G_{R}}{G_{R}^{T} P G_{R}}
$$

Where:

$$
F_{0}=-\left[H+G^{T} P G\right]^{-1} G^{T} P \Phi, \quad \xi=\Phi+G F_{0}
$$

\section{Simulation and Experiment}

In servo control system design, it is very important to design feedback control regulators of prescribed structure for both stability augmentation systems and control augmentation systems. The importance of using servo systems in contrast to open loop systems include the need to improve transient response times, reduce the steady state errors and reduce the sensitivity to load parameters. Improving the transient response time generally means increasing the system bandwidth. Faster response times mean quicker settling allowing for higher machine throughput. Reducing the steady state errors relates to servo system accuracy. Finally, reducing the sensitivity to load parameters means the servo system can tolerate fluctuations in both input and output parameters. Generally, the servo control can be classified into two fundamental classes of problems. The first one addresses the command tracking. It covers the question of how well does the actual motion follow what is being commanded. The model commands in rotary motion control are position, velocity, acceleration and torque. In the linear motion, the force is used instead of torque. The part of servo control that directly deals with this is often referred to as "Feed-forward" control. It can be thought of as what internal commands are needed such that the user's motion commands are followed without any error, assuming of course a sufficiently accurate model of both the motor and load is known. The second general class of servo control covers the disturbance rejection characteristics of the system. Disturbances can be anything from torque disturbances on the motor shaft to incorrect motor parameter estimations used in the feed-forward control. In contrast to feed-forward control, which predicts the needed internal commands for zero following error, disturbance rejection control reacts to unknown disturbances and modeling errors. Complete servo control systems combine both these types of servo control to provide the best overall performance.

\subsection{Motion Controller Design for 3-Axis servo Table}

The schematic of the experiment system is illustrated in Figure 1, and the experimental system hardware environment is illustrated in the Figure 2. The experiment system includes: PC unit, Universal Motion and Automation Controller (UMAC), three units of servo drives, three servo motors, and Three-axis XYZ servo table. Both axes are driven by similar mechanical components. UMAC is linked with servo drive which is linked with matching servo motor; then servo motor drives precision positioning platform through coupling. The signal line of encoder of motor is linked with wiring board corresponding to UMAC. It feeds back the actual position of 
motor by encoder of motor; due to the influence of mechanical transmission, it does not feedback the actual position of platform slider, actually it is a semi-closed loop feedback system, if it is equipped with grating rule, it will be a closed loop feedback system; due to the limit of experimental condition.

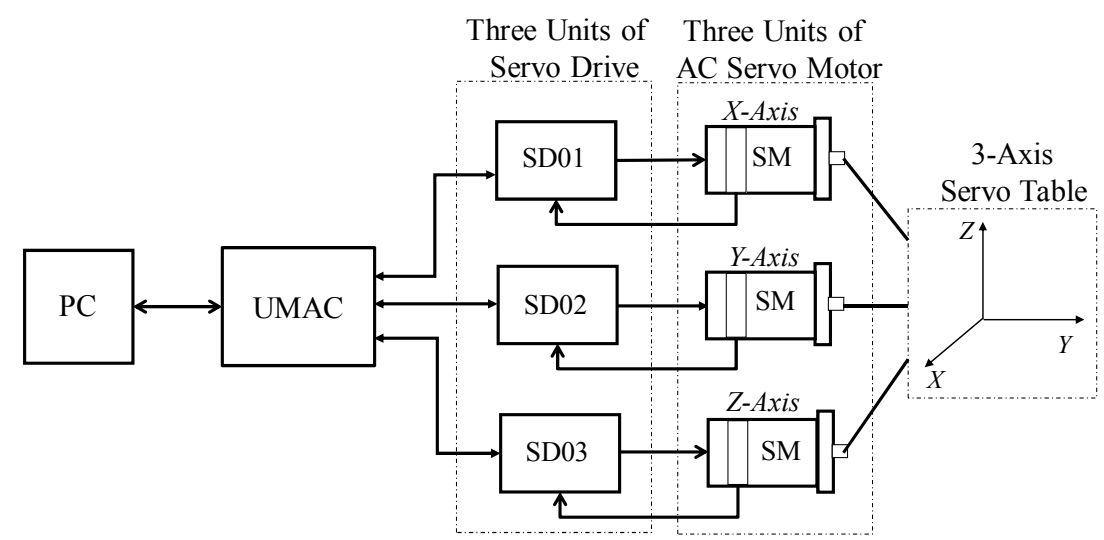

Figure 1. Scheme of the experiment

Ball screws are used to move each load in $\mathrm{X}, \mathrm{Y}$ and $\mathrm{Z}$ directions. The output torque of motor is transmitted to screw rod shaft through couple, and the connection between motor and screw rod of the equipment adopts axial direction rigid coupling, so it can be deemed that there is no transmission error between them. But for the place where screw rod is linked with slider, the above-mentioned acting force appears as radial force $F_{r}$ and axial force $F_{s}$ pushes slider with the load to carry out rectilinear motion along guide rail. However, the radial force $F_{r}$ will produce a driving torque. Due to the inertial moment of screw shaft is relatively small, so we can ignore the influence of screw shaft's rotation on the moment of force.

$$
F_{r} \times r=T ; \quad F_{s}=F_{r} \times \operatorname{ctg} \alpha \Rightarrow F_{s}=\frac{T}{r \times \operatorname{ctg} \alpha}
$$

Where: $\alpha$ : angle of deviation; $r$ : The radius of screw shaft; $T$ : Output torque of motor.

Take slider as an object studied, the equation of motion of screw shaft transmission can be established according to Newton second law:

$$
m \ddot{x}+\beta \dot{x}=F_{s}
$$

Where: $m$ is mass of the load; $\beta$ is the damping coefficient; $x$ is the shift of the Load movement; $\dot{x}$ is the speed $v$ of slider, and $F_{s}$ is the axial force of screw rod on holding frame. The damping coefficient $\beta$ in equation is an important parameter in the establishment of model. It represents the friction condition suffered during the motion of system. This value can be obtained through experiment. Let motor do uniform motion, and then use the monitoring function of servo drive to check instant torque value of motor. According to the above equation of system, it can be converted to the trust size of screw shaft on slider, and it is numerically equal to arithmetic product of speed and damping coefficient. When the motor does uniform motion, its acceleration is zero, that is to say:

$$
\ddot{x}=0 \Rightarrow \beta=\frac{F_{s}}{\dot{x}} \Rightarrow \beta=\frac{T \cdot \operatorname{ctg} \alpha}{r \cdot v},
$$

Table 1 shows the servo system parameters used in the simulation and experiment. 
Table 1. Servo system parameters used in the simulation and experiment

\begin{tabular}{ll}
\hline Type Rating & Value of rating \\
Power of motor $(\mathrm{P})$ & $\mathrm{SMx}, \mathrm{y}=200[\mathrm{~W}], \mathrm{SMz}=100[\mathrm{~W}]$ \\
Rated torque $(\mathrm{T})$ & $\mathrm{Tx}, \mathrm{y}=0.637[\mathrm{~N} \cdot \mathrm{m}], \mathrm{Tz}=0.307[\mathrm{~N} \cdot \mathrm{m}]$ \\
& $\beta \mathrm{x}=22.262[\mathrm{~N} \cdot \mathrm{s} / \mathrm{m}], \quad \beta \mathrm{y}=14.453 \mathrm{~N} . \mathrm{s} / \mathrm{m}], \quad$ and \\
Damping coefficients $(\beta)$ & $\beta \mathrm{z}=12.3 \mathrm{~N} . \mathrm{s} / \mathrm{m}]$ \\
Total Mass $(\mathrm{m})$ & $\mathrm{mx}=26.9[\mathrm{~kg}], \mathrm{my}=14.41[\mathrm{~kg}]$, and $\mathrm{mz}=1.92[\mathrm{~kg}]$ \\
Radius of screw shaft $(\mathrm{r})$ & $\mathrm{rx}=\mathrm{ry}=\mathrm{rz}=7[\mathrm{~mm}]$ \\
Conversion factor $(\mathrm{k})$ & $\mathrm{kx}=\mathrm{ky}=\mathrm{kz}=\mathrm{T} /(\mathrm{r} \times \mathrm{ctg} \alpha) \approx 1.16$ \\
Maximum stroke & $400[\mathrm{~mm}]$ \\
Displacement of target trajectory & $1500[\mathrm{~mm}]$ \\
Screw pitch & $5[\mathrm{~mm}]$ \\
\hline
\end{tabular}

\subsection{Simulation Results and Analysis}

\subsubsection{Simulation Results}

When the slider carries out uniform motion at different speeds, the corresponding torque value is observed under the torque monitoring mode of servo amplifier; in this way, it can find out the relationship between them. The purpose of doing this experiment is to establish a transition between torque and velocity. According to the following data, it can get a similar relationship in MATLAB by data fitting. Table 2 shows a set of data obtained from experiment in accordance with the above principle of experiment. By using MATLAB, and from the above data, we have obtained the following equations:

$$
V_{x}=22.38 \times T_{x}-84.22 ; \quad V_{y}=26.77 \times T_{y}-108.7 ; \quad V_{z}=20.16 T_{z}-86.73
$$

Where: $V_{i}(i=x, y, z)$ represents the velocity of various platforms, while $T_{i}(i=x, y, z)$ represents the torque of various platforms. In this way, it establishes the bridge between torque and velocity, and accordingly provides input data for the following experiment. And According to Eq. 1 and Eq. 2, and when $\mathrm{D}=0$, the specific space model of experimental system is defined as the following:

$$
\dot{X}=A X+B U ; \quad U=C X
$$

Where: $\mathrm{X}$ is the state variables and $\mathrm{U}$ is the input variable defined as the following:

$$
X=\left[\begin{array}{llllll}
x_{p x} & x_{v x} & x_{p y} & x_{v y} & x_{p z} & x_{v z}
\end{array}\right]^{T}, \quad U=\left[\begin{array}{lll}
T_{x} & T_{y} & T_{z}
\end{array}\right]^{T}
$$

$X_{p i}$ : Axis shift; $X_{v i}$ : Axis motion velocity and Ti: Axis input torque; and $(i=x, y, \mathrm{z})$.

Table 3. experimental relation between velocity and torque

\begin{tabular}{llllll}
\hline $\mathrm{T}_{\mathrm{x}}[\mathrm{N} . \mathrm{mm}]$ & $\mathrm{V}_{\mathrm{x}}[\mathrm{cts} / \mathrm{s}]$ & $\mathrm{T}_{\mathrm{y}}[\mathrm{N} . \mathrm{mm}]$ & $\mathrm{V}_{\mathrm{y}}[\mathrm{cts} / \mathrm{s}]$ & $\mathrm{T}_{\mathrm{z}}[\mathrm{N} . \mathrm{mm}]$ & $\mathrm{V}_{\mathrm{z}}[\mathrm{cts} / \mathrm{s}]$ \\
\hline 37 & 743.7 & 32 & 751.8 & 50 & 921.2 \\
70 & 1482.1 & 61 & 1527.3 & 89 & 1707.3 \\
110 & 2377.1 & 89 & 2276 & 130 & 2533.8 \\
147 & 3205.1 & 119 & 3078.2 & 173 & 3400.6 \\
180 & 3943.5 & 151 & 3933.9 & 211 & 4166.6 \\
215 & 4726.6 & 185 & 4843.1 & 265 & 5255.1 \\
\hline
\end{tabular}


With a continue time system and based on the above parameters matrices $\mathrm{A}, \mathrm{B}$ and $\mathrm{C}$ are obtained as the following:

$$
A=\left[\begin{array}{cccccc}
0 & 1 & 0 & 0 & 0 & 0 \\
0 & -11595 & 0 & 0 & 0 & 0 \\
0 & 0 & 0 & 1 & 0 & 0 \\
0 & 0 & 0 & -1281 & 0 & 0 \\
0 & 0 & 0 & 0 & 0 & 1 \\
0 & 0 & 0 & 0 & 0 & -457
\end{array}\right], B=\left[\begin{array}{ccc}
0 & 0 & 0 \\
604 & 0 & 0 \\
0 & 0 & 0 \\
0 & 80.5 & 0 \\
0 & 0 & 0 \\
0 & 0 & 43.1
\end{array}\right], C=\left[\begin{array}{llllll}
1 & 0 & 0 & 0 & 0 & 0 \\
0 & 0 & 1 & 0 & 0 & 0 \\
0 & 0 & 0 & 0 & 1 & 0
\end{array}\right]
$$

Using MATLAB to discrete the system with $0.2 \mathrm{~s}$; target trajectory is derived as spiral form:

$$
R(i)=\left[\begin{array}{c}
R_{x}(i) \\
R_{y}(i) \\
R_{z}(i)
\end{array}\right]=\left[\begin{array}{c}
0.15 \cos (t) \\
0.15 \sin (t) \\
0.1 t
\end{array}\right]=\left[\begin{array}{c}
0.15 \cos (0.2 i) \\
0.15 \sin (0.2 i) \\
0.1(0.2 i)
\end{array}\right]
$$

Weighting factors choosing as the following;

$$
\begin{aligned}
& Q=\operatorname{diag}\left(\left[\begin{array}{lllllllll}
1000 & 1000 & 1000 & 10 & 10 & 10 & 10 & 10 & 10
\end{array}\right)\right. \\
& H=\operatorname{diag}\left(\left[\begin{array}{lllllll}
0.01 & 0 & 0 ; 0 & 0.01 & 0 ; 0 & 0 & 0.01
\end{array}\right]\right)
\end{aligned}
$$

By using MATLAB Figure 2 shows the simulation results of the position response and the tracking error of the 3 -axis servo table system. Take the above velocity as input to obtain the actual trajectory, and adopt PVT motion mode to achieve; the PVT mode algorithm of UMAC cards is unique and effective as it just needs specified time, specified terminated velocity to realize the control of specified velocity through the positions calculated by specified formula. Because the UMAC uses the control in the way of pulse plus direction, it fits precision positioning platform and its biggest advantage is precision positioning; before the pulse command is issued, it will calculate the trajectory of spline by DSP processor of UMAC in advance to ensure the smooth transition between spline curves, so no matter what control method we adopt, its trajectory is smooth curve.

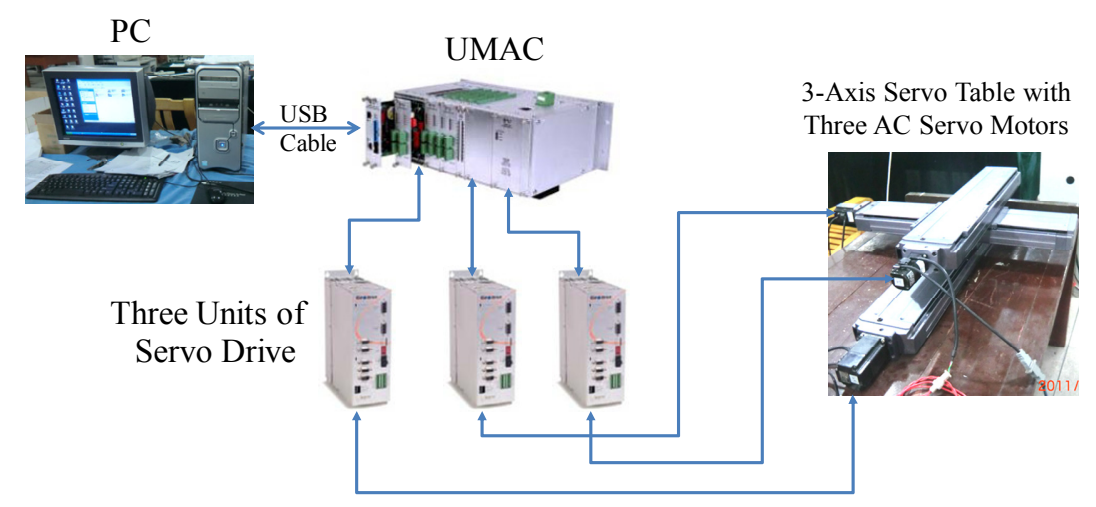

Figure 2. Experimental system hardware environment

\subsubsection{Simulation Analysis}

We can see that the simulation trajectory fits object trajectory well, and almost coincides with it. Normal direction error synthesizes the error of various axial directions, and it evaluates the whole tracking error of space object trajectory. It can be seen from the Figures 3 and 4 that the simulation for spiral form gets good effect, from its normal error analysis chart, it can be seen that its maximum error is not more than $0.3 \%$, and it has got good effect on the whole. Then carry out analysis on various axes. It can be seen from the error analysis of various axes that, except there is an abnormity in the place close to 0 position. According to the error analysis of $\mathrm{X}$-axis, it can be seen that it does not have laws, but there are some mutations in some place, then its error value stabilized at about $1 \%$. But according to the error analysis chart of Y-axis, it can be seen that it reached up to $4 \%$ 
at the beginning, while it gradually decreased and then stabilized, which is a normal phenomenon. From the simulation chart of $\mathrm{Z}$-axis, it can be seen that it reached up to $1.2 \%$ at the beginning and then its error value is close to zero. The above adopts optimal preview tracking control algorithm to carry out tracking simulation for space object trajectory, and it makes use of optimal preview tracking control algorithm to track the space objects trajectory, and obtains good results. It can summarize the following conclusions according to the simulation analysis:

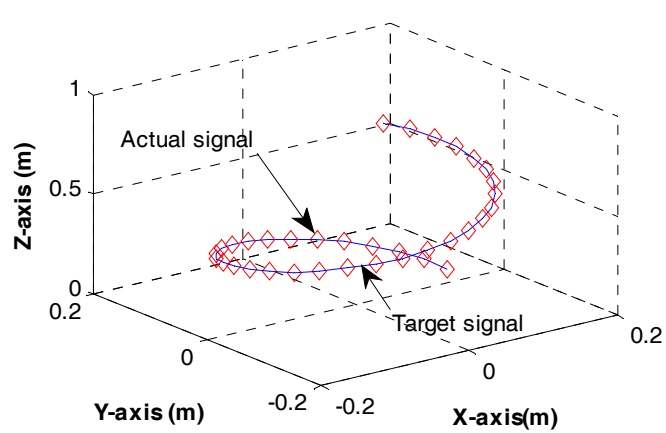

(a)

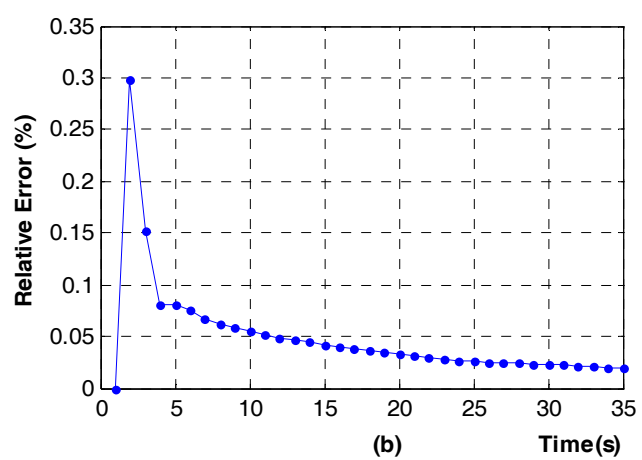

(b)

Figure 3. (a) response of the 3-axis for spiral trajectory (b) relative tracking error of spiral trajectory
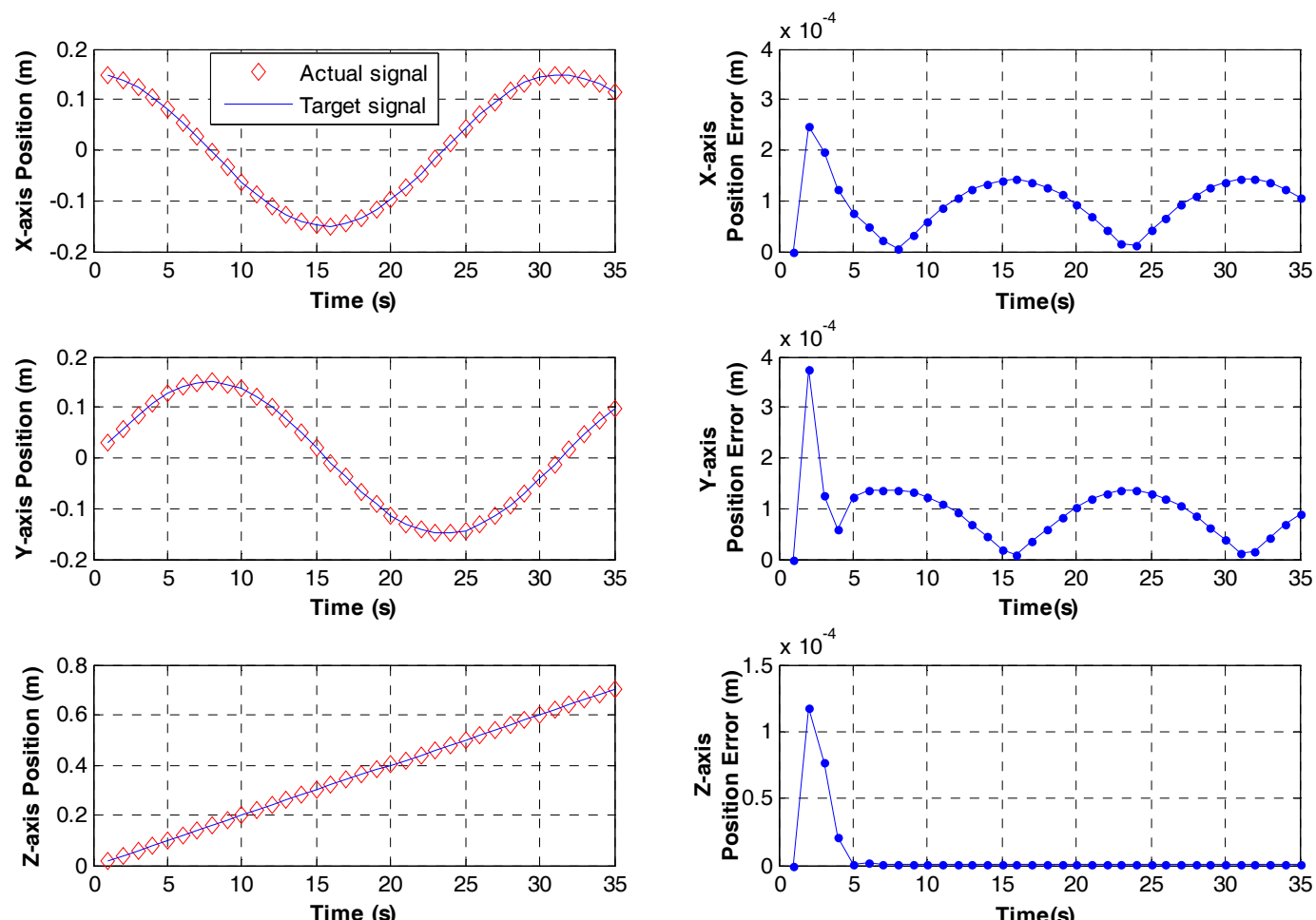

(a)

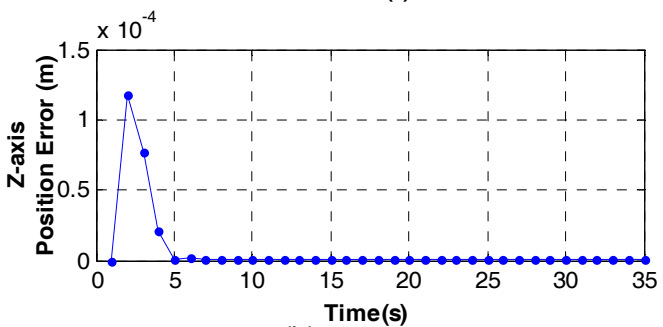

(b)

Figure 4. (a) response of the 3-axis spiral trajectory, (b) tracking error

- Making use of optimal preview tracking control algorithm to track the space object trajectory, and obtain good results, which indicates that the optimal preview tracking control algorithm is sufficiently effective.

- From the simulation stage, it seems to get great success. The essence of simulation is simulating the actual trajectory, i.e. an output, and there must be an input corresponding to an output, that is to say there is an input condition to get this good result. When giving this input, the actual trajectory of experimental platform may not coincide with the simulation trajectory. It may be along the course from input to output, that is to 
say, the mathematical model established deviates to some extent. Therefore, it is very necessary to conduct experimental research, since practice is the only criterion for testing the truth.

- From the previous analysis, it can be known that, obtaining this simulation results are dependent on many factors, such as, choice of matrix $Q$ and $H$, and determination of preview steps, which have a significant impact on simulation results. Therefore, the correct arrangement of parameters is also an important factor in getting simulation. Simulation is the premise for carrying out experimental study, and the simulation results provide a basis for subsequent experimental research.

\subsection{Experiment Results and Analysis}

Download the compiled experimental program to UMAC card, the data collection graph after execution is obtained: because UMAC card's output is pulse, its unit is expressed by pulse. Velocity unit is cts/s and position unit is cts. Its conversion is: $5 \mathrm{~mm}$ per 2048 pulses. From the data sample graph, it can be seen that the position curve of each axis is smoother, the middle part of it is closer to straight line, and the turning point of each axis is smoother. Velocity is not very stable, which can be seen in the actual motion. But in general, it is a curve like sine and cosine (the reciprocal of the position), which is not very far off reality. The reason may be that the adjustment of motor controller parameter is not very satisfactory.

In the velocity sample graph which is illustrated in figure 5, for each axis; $X, Y$ and $Z$ it is obvious that the amplitude of fluctuation at the starting point of velocity is wide, which is because the response process of motor. From the sample graph of position which is illustrated in Fig 6, it can be seen that the position curves of each axis are similar to the sine and cosine curve. The shape of curve shows that experimental trajectory is quite consistent with simulation and the object trajectory. The velocity curve of each axis is not a smooth curve, and it is also not smooth in the actual motion, because the experimental input is a constant velocity in each equal time interval, and it has a transition process on the speed in the next time interval, therefore, it cannot be a smooth curve. But in general, it maintains a derivative relationship with position curve. It also has a certain relationship with the specified parameters of motor, so if the configuration of parameters is proper, its trajectory is a smooth curve.

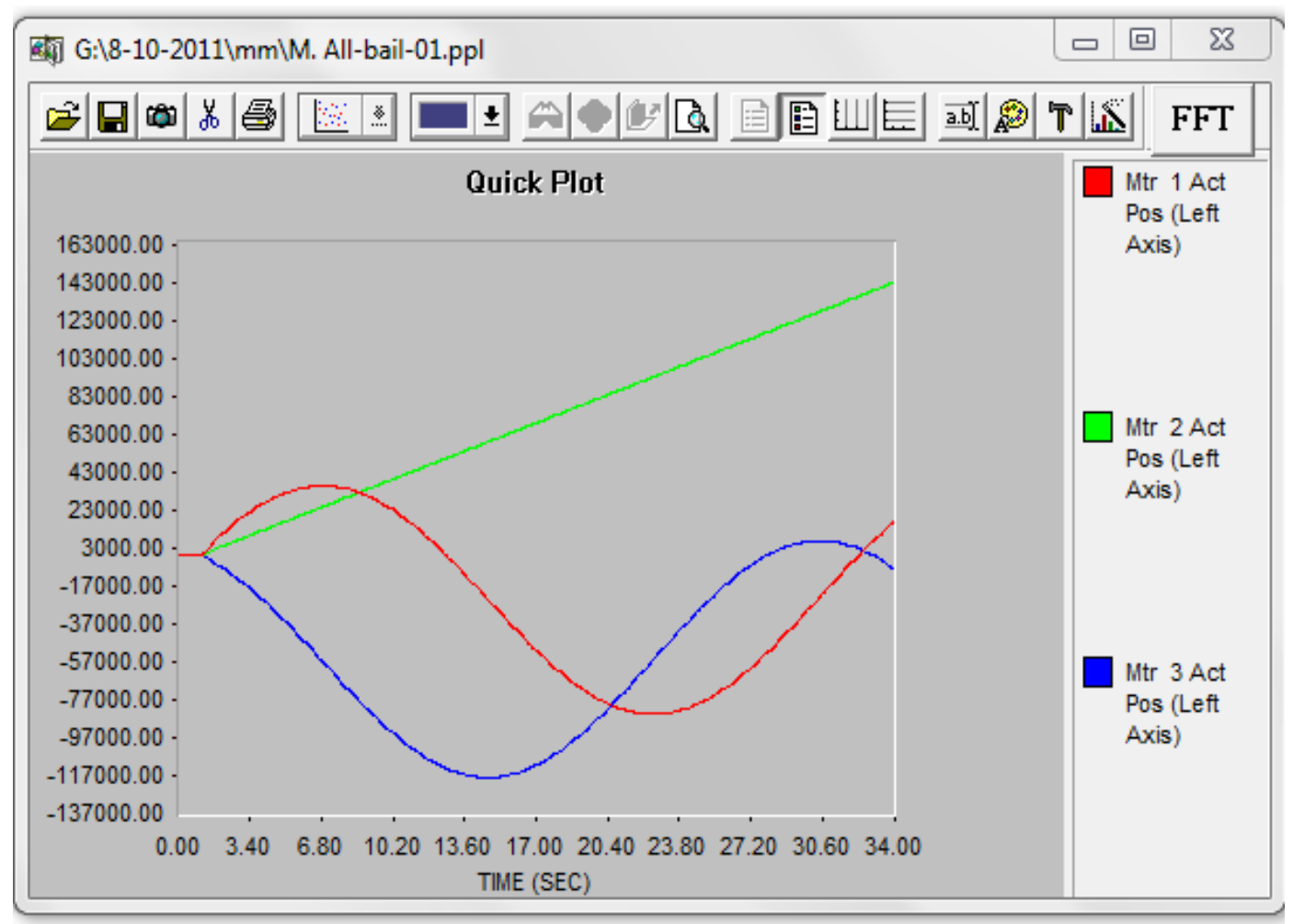

Figure 5. Experimental Velocity response 


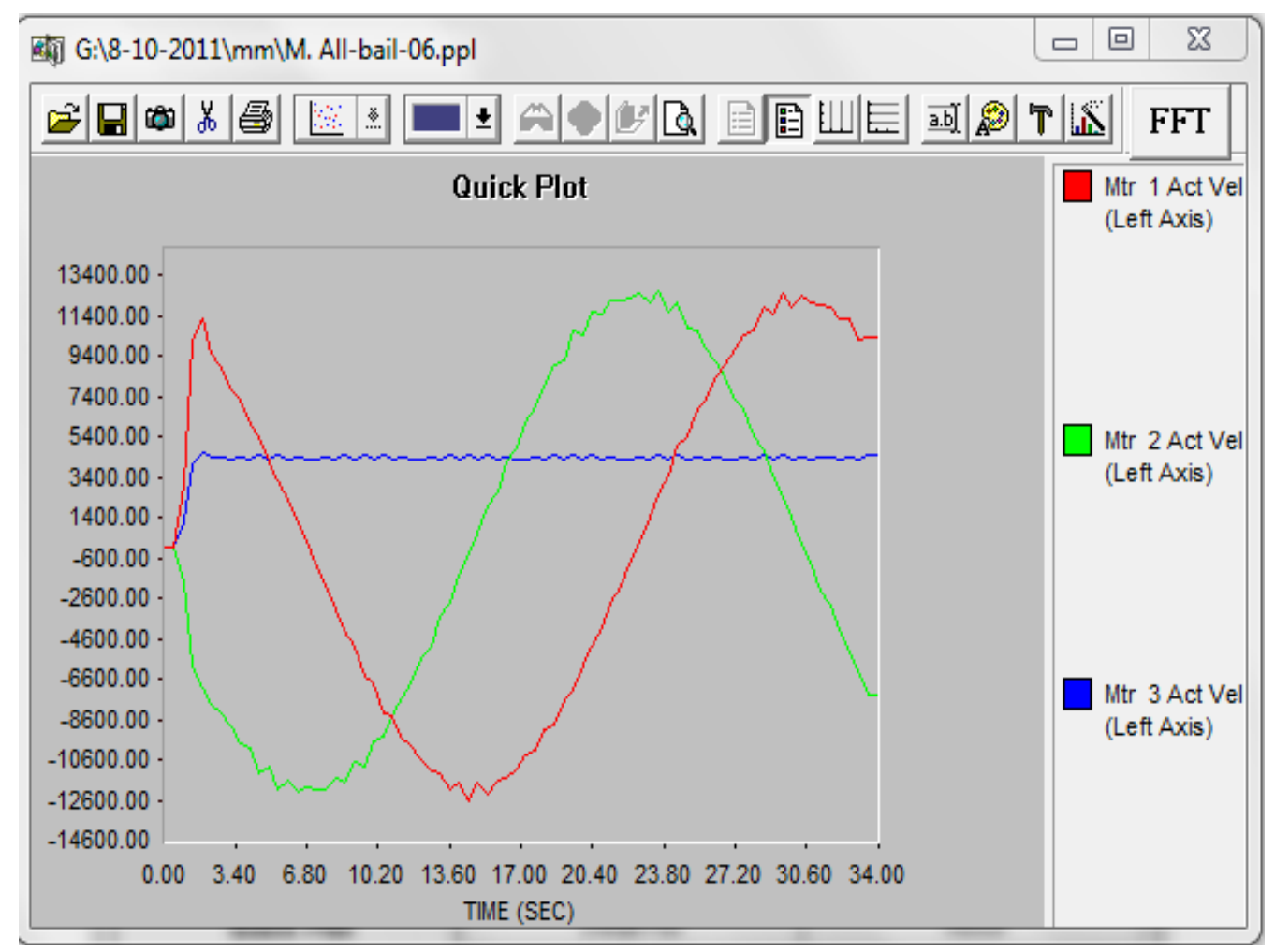

Figure 6. Experimental Position response

\section{Conclusion}

Optimal preview control system can be constructed from the idea to target value. It is similar to the result of trying to shape a better target for reply the human intuition.

Through the simulation research of algorithm, it shows that the proposed optimal preview control algorithm is effective, but also it shows that the optimal preview tracking control algorithm has been very mature in the linear space theory except for that there is a deviation occurred in the process of establishing experimental model.

Through experimental analysis, it verifies that there is a certain deviation between simulation and experiment, the experiment is the final inspection for tracking control algorithm, although the experimental result has large error, yet it is an effective tracking control algorithm. Combination of theory and practice will reflect a great vitality, so it must also strengthen the applied research of optimal preview tracking control algorithm.

When the previews steps are zero, not found, then we have no feed forward compensation. Such a control system is optimal control system OCS yet it is not an optimal preview control system OPCS. That is to say that optimal control system OCS is a special case of optimal preview control system OPCS when preview step are zero.

Optimal preview control method for servo systems is an effective to improve the phase response as compared to optimal control method. The future information has great importance in determining human behavior. Hopefully, use of the technology depending on future information will increase extensively in different areas.

\section{Summary}

In the optimal control system for tracking system, a feed-forward compensator will be added to the system to be more able to trace, along with maintaining a good stability. The goal is to follow a certain specified trajectory. Therefore, a feasible reference trajectory is assumed to be given i.e., a trajectory that can be realized for the system under consideration. The tracking error at each time is defined as the difference between "where the system is" and "where it should be". The problem, now, is to design a controller for the system for which the tracking error converges to zero.

\section{References}

Li, D., Zhou, D., Hu, Z., \& Hu, H. (2000). Optimal Preview Control Applied to Terrain Following Flight. in Proc.
IEEE
Conference
on Decision
and
Control,
Orlando, Florida,
211-216. 
http://dx.doi.org/10.1109/.2001.980100

Li, Bin, \& Lu, Hailong. (2011). Application of Preview Control in Linear Motor Servo Mechanism. IEEE. 4778-4781. http://dx.doi.org/10.1109/MACE.2011.5988080

Andrew, Hazell. (2008). Discrete-time optimal preview control. London: Imperial College University of London

Chandeok, Park, \& Daniel, J., Scheeres. (2006). Solving Optimal Feedback Control Problems by the Hamilton-Jacobi Theory. Proceedings of the 2006 American Control Conference Minneapolis, Minnesota, USA, IEEE, 2406- 2411. http://dx.doi.org/10.1109/ACC.2006.1656580

Sharp, R. S., \& Valtetsiotis, V. (2001). Optimal Preview Car Steering Control. ASME Journal of Vehicle Systems Dynamics (Supplement), 35, 101-117.

Ren, Z., Chen, J., hara, Shinji, \& Qiu, L. (2001). Optimal Tracking Performance: Preview Control and Exponential Signals. IEEE Trans, Autom, Control, 39(46) 1647-1654. http://dx.doi.org/10.1109/CDC.2000.912144

Lewis, F. L., \& Syrmos, V. L. (1995). Optimal Control. John Wiley \& Sons. ISBN 0-471-03378-2.

Sheridan, T. B. (1966). Three Models of Preview Control. IEEE Trans. on Human Factors in Electronics, HFE-7(2), 91-101. http://dx.doi.org/10.1109/THFE.1966.232329

Bender, E. K. (1968). Optirnum Linear Preview Control with Application to Vehicle Suspension. Trans. of ASME, J. of Basic Engineering, 213-221. http://dx.doi.org/10.1115/1.3605082

Tomizuka, M. (1976), Optimum Linear Preview Control with Application to Vehicle Suspension - Revisited. Trans. of ASME, J. of Dynamic Systems, Measurement, and Control, 98(3), 309-315. http://dx.doi.org/10.1115/1.3427040

Liao, F. C., Cui, Y. H., \& Shen, Z. W. (2009). Optimal preview control for linear time-variant discrete systems. International Conference on Machine Learning and Cybernetics, 4, 1954-1960. http://dx.doi.org/10.1109/ICMLC.2009.5212208

Lin, F., \& Brandt, R. D. (1998). An optimal control approach to robust control of robot manipulators. IEEE Transactions on Robotics and Automation, 14(1), 69-77. http://dx.doi.org/10.1109/70.660845 